\title{
Vigilancia epidemiológica de la intoxicación aguda en el área sur de la comunidad de Madrid: estudio Veia 2004
}

\author{
P. J. CABALLERO VALLÉS' ${ }^{1}$, S. DORADO POMBO, A. DÍAZ BRASERO, M. \\ EUGENIA GARCÍA GIL, L. YUBERO SALGADO, N. TORRES PACHO, C. IBERO \\ ESPARZA, J. CANTERO BENGOECHEA
}

${ }^{\prime}$ Centro de Salud "Ángela Uriarte”. Hospital Universitario 12 de Octubre. Madrid

EPIDEMIOLOGIC SURVEY OF ACUTE POISONING IN THE SOUTH

AREA OF THE COMMUNITY OF MADRID. THE VEIA 2004 STUDY

\section{RESUMEN}

Objetivo: VEIA es un registro evolutivo de las intoxicaciones agudas (IA) atendidas en Urgencias del Hospital Doce de Octubre de Madrid en un año completo [19791 (2), 1985 (3), 1990 (4), 1994 (5), 1997 (6) y 2000 (7)] presentamos el estudio del año 2004 y comparamos los resultados con los de años anteriores (1-7).

Métodos y resultados: el método es idéntico. De las 1.508 IA, 610 son intentos de suicidio (IBAIS) 319 etílicas (IAVE) y 219 drogas (IAVD). De los 2259 tóxicos implicados el $48 \%$ son medicamentos (50\% diazepóxidos) alcohol 25\% y drogas el $13 \%$.

Conclusiones: este año ha supuesto un importante aumento (34\%) del número de casos y la incidencia supera a todas las publicadas en nuestro país $(25,31,40)$ Aunque en el conjunto no hay diferencia significativa de género con el Censo de Área, la hay en IBAIS, IAVE y IAVD Las IBAIS crecen un 35\%; Se duplica el uso de benzodiacepinas y antidepresivos en los IS de mujeres y crecen también en hombres pero menos; El paracetamol se mantiene en el 23\% AINES, adyuvantes y mío-relajantes aumentan en mujeres y también el uso de alcohol y otros no fármacos en los is de mujeres casi igualándose con los hombres. La cuarta parte de los hombres con IS eran adictos y aparecen 13 casos de trastornos alimentarios. En las IAVE crecen el grupo sin etilismo y baja el total. Las drogas se duplican con respecto al año anterior la cocaína supone los 2/3 también suben la MDMA (22 casos) y aparecen nuevas drogas, como el pegamento, sin duda efecto de la inmigración, y ketamina; incluso cuatro casos no pudieron ser etiquetados por la falta de medios diagnósticos en la urgencia.

PALABRAS CLAVE: Intoxicación aguda. Epidemiología. Intentos de suicidio. Intoxicación medicamentosa. Intoxicación alcohólica. Intoxicación por drogas. Intoxicación accidental.
ABSTRACT

Objetive: VEIA study is an evolutional registry of acute poisonings (AP) attended in the Emergency Room of the Doce de Octubre Hospital in Madrid (Spain) in a whole year (1979,1985, 1990, 1994,1997 and 2000). We present the 2004 study and compare the results with the previous years.

Methods and results: Methodology has been identical across VEIA STUDY. Of 1508 AP, 610 are suicide attempts (IAVIS), 319 ethylic, (IAVE), and 218 by illicit drugs (IAVD). Of the 2,259 toxics involved, $48 \%$ are medications (50\% benzodiacepines) alcohol $25 \%$ and illicit drugs $13 \%$.

Conclusions: There is an important increase (34\%) of cases and the incidence surpasses all published in our country. There are no gender differences nor in the whole neither the Health Area Census, but there are differences in IAVIS, IAVE and IAVD. IAVIS increase in 35\%. Benzodiacepines poisoning increases two-fold as well as antidepressive drugs do in women. In men also increase, but in a minor extent. Acetaminophen remains the same in 23\%. NSAID's, adjuvants and myorelaxants increase in women as do also alcohol and other poisons that almost equal men's. There are 13 cases of IAVIS in patients with alimentary disorders. Among men, a quarter are illicit drug abusers. In IAVE, the group without alcoholism grows and the total decreases. Illicit drugs duplicate the number of the former year. Cocaine supposes already 2/3 of the cases, MDMA ascends to 22 cases and they appear new substances as glue, without doubt as an effect of immigration and ketamine. Finally 205 household accidents and 57 industrial injuries complete the series.

KEY WORDS: Acute intoxication. Epidemiology. Suicide attempts. Medicine intoxication. Alcohol intoxication. Drug intoxication. Acute poisoning.

Caballero Vallés PJ, Dorado Pombo S, Díaz Brasero A, García Gil ME, Yubero Salgado L, Torres Pacho N, Ibero Esparza C, Cantero Bengoechea J. Vigilancia epidemiológica de la intoxicación aguda en el área sur de la comunidad de Madrid: estudio Veia 2004. An Med Interna (Madrid) 2008; 25: 67-72.

\section{INTRODUCCIÓN}

El Estudio Veia examina la IA desde 1979 mediante cortes cuatrienales que recogen todos los casos asistidos en Urgencias del Hospital Universitario 12 de Octubre durante un año (1-7) En el presente artículo exponemos los resultados de 2004. Justifican este importante esfuerzo las grandes variaciones que, el modo de intoxicarse, sufre con el paso de los años instigados por factores sanitarios, farmacológicos o sociales. IA por nuevos medicamentos y drogas disponibles pero también usos diferentes de tóxicos clásicos y sobre todos variaciones en los hábitos sociales son las diferencias esperadas (124) Por otra parte a lo largo de estos años otros muchos estudios realizados en nuestro país ha evidenciado que nuestros resultados reflejan de modo fidedigno la cambiante realidad de las IA en España $(9,14,15,18,19,24-40)$. 


\section{MATERIAL Y MÉTODO}

Se han recogido y estudiado los casos de IA atendidos en la Sección de Urgencias del Hospital Universitario 12 de Octubre de Madrid durante el año 2004. Este Centro ofrece asistencia sanitaria al Área 11 del SERMAS y es referencia de otros centros por lo que asiste a 643.740 personas mayores de quince años (41). El número de urgencias médicas atendidas fue 186.168 médicas y de ellas 1.508 correspondieron a IA. La metodología es igual a la de anteriores publicaciones (1-7) por lo que denominamos intoxicación al contacto de productos tóxicos potenciales: a) medicamentos: cuando superan la dosis máxima terapéutica, incluso si intentan mitigar un síntoma y se ingiere una cantidad excesiva (sobredosificaciones); b) alcohol y drogas: cuando interfieren una actividad mínimamente normal, por ejemplo el equilibrio; y b) otros tóxicos, a cualquier dosis. Dividimos las IA en voluntarias: (IAV), a su vez repartidas en intentos de suicidio (IAVIS) etílicas IAVE, drogas IAVD y sobredosificaciones (IAVSD), según la intención y accidentales (IAA): a su vez laborales (IAAL) o domésticas (IAAD), dependiendo del lugar donde ocurren. Excluimos las toxiinfecciones alimentárias y las iatrogénicas. Los tóxicos se clasifican en: medicamentos y no medicamentos. Hablamos de fármacos o medicamentos para referirnos a los principios activos y de presentaciones farmacéuticas para denominar los distintos tipos de preparados comerciales empleados por cada paciente que pueden ser asociaciones o no.

Hemos analizado la distribución de los casos según la edad, sexo, tipo de intoxicación y tóxico. También se han estudiado los antecedentes, así como su posterior derivación y la adscripción o no del paciente al Área de cobertura del hospital. Se trata de un estudio descriptivo transversal en el que se han empleado técnicas básicas de estadística descriptiva. Cada grupo se define por la proporción sobre el total en variables cualitativas y la media, mediana y/o moda, así como desviación estándar para las cuantitativas. Para la comparación de las medias se utilizó el Chi cuadrado y la prueba de Student, el límite de significación se consideró $\mathrm{p}<0,05$ y el intervalo de confianza del $95 \%$.

\section{RESULTADOS}

\section{CARACTERÍSTICAS GENERALES}

Se atendieron 1.508 IA (234/100.000 habitantes), correspondientes al $1,77 \%$ de las urgencias médicas y el $0,68 \%$ de las totales. El número de mujeres es 756 (50\%) y 752 los hombres (50\%), con una proporción M/H de $1: 1$.

Las edades oscilaron entre 15 y 96 años, con una media \pm desviación estándar para el conjunto de la serie de $36 \pm 15$ años (mediana y moda 33), $36 \pm 14$ para los hombres (mediana 35 , moda 34 ) y $36 \pm 16$ para las mujeres (mediana 32, moda 36); la diferencia es significativa al comparar la edad media entre los sexos (error estándar: 0,91 p < 0,05). La distribución por edades y género, así como la pirámide poblacional del Área 11 de según los datos de la Memoria del Hospital se expone en la figura 1. Un $20 \%$ de los pacientes atendidos no pertenecían a la zona de influencia del Hospital.

\section{TIPO DE INTOXICACIÓN}

El número de casos de los diferentes tipos de intoxicación, su género, así como la media y la desviación estándar de las edades, figuran en la tabla I.

- Intoxicaciones agudas voluntarias (IAV) (150/100.000 habitantes). El reparto por edades en cada tipo de IAV se muestra en la figura 2. Las medias de edad de uno y otro sexo en el conjunto de IAV son significativamente diferentes (error estándar: $0,90, \mathrm{p}<0,001)$ y lo mismo cabe decir en las intoxicaciones alcohólicas (error estándar: 1,53, p < 0,001), y las IAV por drogas $\mathrm{p}<0,05$, pero no en los restantes subgrupos.

- Intoxicaciones accidentales (IAA) (42/100.000 habitantes). Los datos sobre edad y género se exponen en la figura 1.

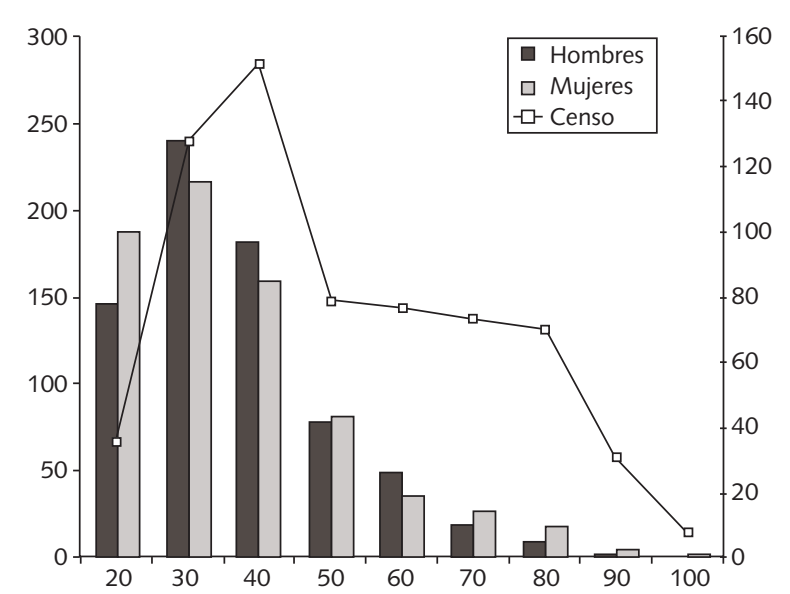

Fig. 1. Reparto numérico por edad y género.

\section{TÓXICOS IMPLICADOS}

-Medicamentos: tóxico único en 540 casos (36\%) en el grupo general, que asciende hasta el $74 \%$ en los intentos de suicidio (IS) (81\% en las mujeres y $59 \%$ en los hombres). Los fármacos, su reparto conforme a los diferentes grupos terapéuticos y la incidencia según el género pueden verse en la figura 2. Promedio de fármacos por presentación farmacéutica: 1,05.

- No medicamentos: 811 casos 54\% (Fig. 3).

- Asociaciones de ambos 154 casos (10\%).

-Desconocido: 4 casos, $0,2 \%$.

\section{DISTRIBUCIÓN EN EL TIEMPO}

Ni el conjunto de la serie, ni los diferentes subtipos muestran diferencias significativas entre los días de la semana ni los meses del año.

Antecedentes: Un $88 \%$ de los pacientes con IS fueron valorados por un psiquiatra en la Urgencia, obteniéndose 448 antecedentes, todos se enumeran en la figura 4.

\section{DISCUSIÓN}

-Incidencia: Con respecto al 2000 (6) aumentan tanto el número de urgencias $(10.5 \%)$ como el de IA $(33,7 \%)$ pero la 


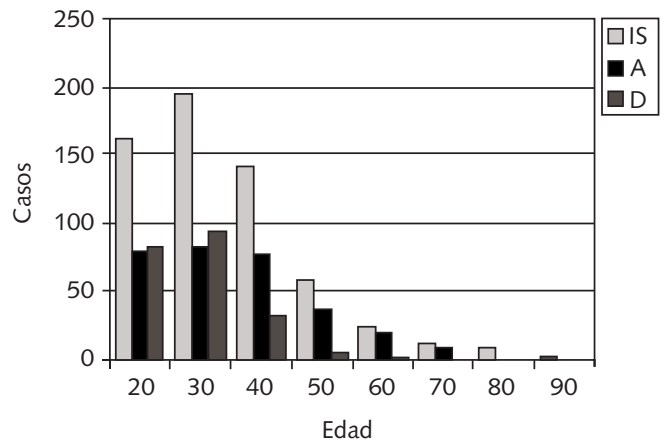

Fig. 2. Distribución por grupos de edad IAVIS, IAVD e IAVE.

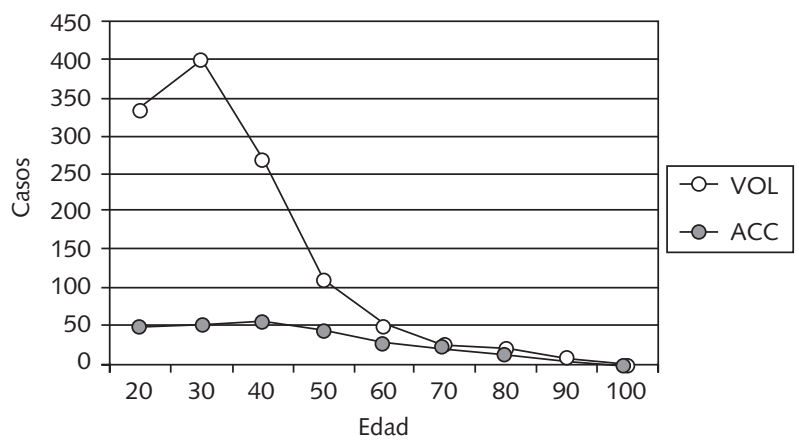

Fig. 3. Distribución por grupos de edad IAV e IAA.

$\chi^{2}$ no muestra asociación entre ambos $\mathrm{p}<0,001$. No hemos encontrado publicaciones que avalen este crecimiento. La incidencia actual (234/\%ooo habitantes y año) es un $34 \%$ mayor que la precedente (170\%ooo) (6) y supera a todas las publicadas en nuestro país $(24,33,38,42)$ pero admitimos que este cotejo puede no ser muy real. Confrontar la incidencia con otras series es tarea difícil porque los diferentes autores recogen grupos de IA no homogéneos y más o menos selectivos (40) (IS sólo por medicamentos, intoxicaciones alcohólicas incluidas o no drogas, edad pediátrica, etc.); En otras ocasiones el Censo Sanitario es confuso y por último, en este tipo de trabajos, obligatoriamente retrospectivos, existe el posible sesgo de una recogida incompleta de casos. La población cubierta por el Hospital Universitario 12 de Octubre, según la Memoria 2004 (41) es 536.450 usuarios, pero si tenemos en cuenta que los Servicios de Urgencia hospitalarios atienden un número no desdeñable de personas no adscritas, que en esta serie se cuantifica entorno a un $20 \%$, debemos concluir que nuestra población cubierta real es de 643.740 personas.

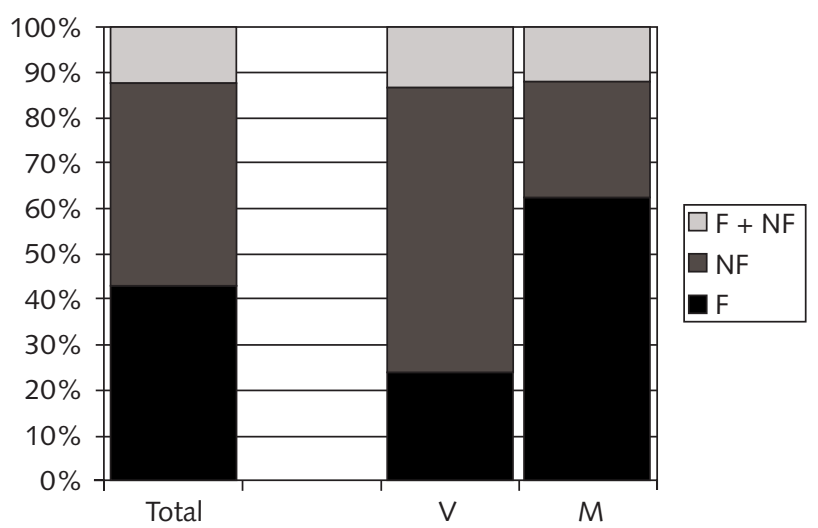

Fig. 4. Tóxicos: repartos según fármacos, no fármacos y ambos.

-Edad y género: Al igual que en las últimas cuatro series (4-7) no hay diferencia de genero, ni considerado el conjunto de las IA, ni con la población de referencia, ni tampoco en las IAV o las IAA en su conjunto. Pero tanto en las IVAIS, IAVE, IAVD, como IAAD o IAAL sí que hay diferencia significativa de género tanto en la comparación con el Censo del Área (48) como, lógicamente, con el grupo general ya que entre ellos no difieren. Lo mismo cabe decir cuando estudiamos los repartos por grupos de edad en los diferentes tipos de IA. Ver Cuadros.

-IVAIS: 610 casos (95\% oooo) 40\% del total (81\% de las mujeres, 59 de los hombres). En los 25 años que hemos estudiado las IAVIS (1-7) los casos han aumentado en ambos sexos, tanto, que en este año, representan casi el doble de 1990 (3) y este crecimiento es mayor que el experimentado por el conjunto de las IA (de 851 a $1.508-177 \%-\chi^{2}=44,4 \mathrm{p}<$ $0,001)$. Esta incidencia es muy superior a las publicadas en los últimos diez años (4-6) pero podría ser una tendencia reciente. En un estudio evolutivo, similares al nuestro, Rygnestad (16) observa un declinar entre 1987 y el 2002 tanto en hombres como en mujeres, pero la OMS (12) alertó de un previsible crecimiento.

-Tóxicos en IAVIS: 1.144 tóxicos se han visto involucrados en estos 610 casos, lo que supone un promedio de 1.87. Algo mayor que otras publicadas (43) Los medicamentos estuvieron implicados en el $84 \%$ de las IAVIS, es habitual que predominen y más en una zona geográfica como la nuestra, urbana con muy poco peso rural $(8,11-13,16,17)$ Los psicofármacos están implicados en el $57 \%$ de las IAVIS y en el $33 \%$ de toda la serie. Entre ellos las más usuales son las benzodiacepinas con el $48 \%$ de toda la serie y el $70 \%$ de las IVAIS, la mayoría de los autores avalan estos datos (18,37-40) El uso de medicamentos en las IAVIS reflejan el consumo de esos medicamentos $(14,21-23,43)$. En España, al igual que en la mayoría de los países industrializados, el consumo de tranquilizantes ha experimentado un importante aumento desde 2002, en torno al 5\% anual hasta el 2005, momento en que se modera ligeramente (45). Las recetas extendidas a mujeres duplican las de los hombres y ellas entre los 45 y 64 años son las máximas consumidoras (45). De acuerdo con estos datos 
el consumo de tranquilizantes habría crecido un $25 \%$ en el intervalo del estudio actual 2000-4 Sin embargo en nuestro estudio el crecimiento supera el 50\% (pasa de 302 casos a 459) las mujeres fueron más proclives con casi las $3 / 4$ partes También los IS con benzodiacepinas crecieron en otros países: $20 \%$ en 1978, 39 en 1987 en Noruega (17) aunque en este caso unos años mas tarde bajaban hasta el $20 \%$. Un debate clásico $(11,21,22,46,47)$ hacía pensar que aumentarían los antidepresivos y disminuirían los tranquilizantes, pero no parece cumplirse $(24,47)$ Dentro del grupo de psicotropos, los antidepresivos ocupan el segundo lugar con el $18 \%$ lo que representa un aumento con respecto al $12 \%$ de los años anteriores $(4-6)$ y a otros publicados en IS $(30,37,48)$. El tratamiento antidepresivo puede disminuir los IS $(8,9,11)$ aunque no está suficientemente estudiado. Los ISRS parecen haber sustituido a los tricíclicos sin que hayamos observado diferencia de género. Los restantes psicofármacos: neurolépticos, barbitúricos y otros, sólo suponen el $10 \%$ de este grupo. El grupo de analgésicos (14\%) aumenta un $20 \%$ con respecto a 2000 (6) sobre la base del aumento cada uno de sus componentes y más concretamente los no narcóticos; el paracetamol asciende hasta el $23 \%$ de los analgésicos permaneciendo aún muy lejos del $43 \%$ en el Reino Unido (11); Aspirina y AINES se mantienen y los restantes se diversifican con varios casos de adyuvantes y mío relajantes. Los analgésicos narcóticos menores están poco representados. No Fármacos $(N F)$ : En 1979 (1) sólo un $0.2 \%$ de los IAVIS fueron por NF, en 2004 son el $7 \%$ El origen es la creciente influencia del rol masculino, pues mientras el $81 \%$ de las mujeres usan fármacos únicamente, los hombres solos en el 59\% De modo antitético; $13 \%$ de los hombres usaron NF solos en su IAVIS, frente al $4 \%$ de las mujeres. El uso de asociaciones de fármacos y no-fármacos es también un modo de actuación masculino $(\mathrm{p}<0,001)$ Es mas, las mujeres usan tanto los fármacos que aunque los asocien y se tenga en cuenta esta asociación también existe diferencia significativa con los hombres (como hemos dicho, ellos usan más asociaciones y parten de un número menor de fármacos) De los IAVIS por NF destaca el etanol, con un $21 \%$ de los casos (solo o asociado); las drogas en un 5\% y los restantes venenos $3,6 \%$ (4 casos de IS con gases, 10 con cáusticos y los 8 restantes corresponden al grupo de miscelánea. Un $24 \%$ de los IS utilizaron asociaciones de F y NF, cifra intermedia respecto al $20 \%$ publicado por Duce et al (43) y al $36 \%$ de Verstraete y Buylaert (57) Un 80\% de estos casos incluían alcohol como NF, en línea con otros estudios (37) Un $20 \%$ restante ha sido drogas y venenos tradicionales. La vía oral ocupa el $95 \%$ de los casos, respiratoria en un $4 \%$ y parenteral en solo 5 casos. No falleció ningún paciente de las IAVIS en este año. La mortalidad, al menos hospitalaria, de este tipo de IA es baja o nula $(12,14,16,17,23,46)$ según los estudios. La mortalidad extrahospitalaria ha sido poco estudiada en nuestro país, pero si nos atenemos a los datos publicados por Lardeur y cols. (42) entre los años 97-99 encontró un 1\% en Vienne (Francia) Tipología de las IAVIS: Se han recogido 448 antecedentes, cifra mayor que otras publicadas $(17,20,54)$. En la valoración psiquiátrica (realizada al $88 \%$ sin diferencia de sexo) durante el ingreso se diagnosticó depresión al 32\% de los pacientes con IAVIS, las tres cuartas partes mujeres, como es habitual, por otra parte ya que en ellas los antecedentes psiquiátricos más comunes son los trastornos emocionales y adaptativos (49). El 11\% admitieron intentos previos, cifra muy inferior a los 18 habituales $(5,6)$ y a otras publicadas
(25), pero este año tenemos el triste record de un paciente con trece intentos previos. Las toxicomanías, tanto alcohol como drogas, en los varones son un importante factor de riesgo para el IS (61-63) y lo mismo cave decir de la esquizofrenia (49), en la presente serie $24 \%$ eran adictos a drogas o alcohol. Está establecido que el $40 \%$ de los alcohólicos lo intentan (52-53) y es más frecuente en los mayores de 50 años que viven solos, parados, y con aislamiento social $(60,61)$. La modificación de éstos factores pueden ser considerados factores protectores frente al suicidio de los alcohólicos $(53,54)$. Trece de los IAVIS se produjeron en pacientes con trastorno alimentario, la misma cantidad que de esquizofrenia o trastornos adaptativos. Además embarazo, ludopatía o malos tratos son circunstancias a tener en cuenta junto con las patologías orgánicas, como la diabetes. Destino de los IS: $1 \%$ ingresó en planta y $2 \%$ en UCI ( 9 mujeres y 2 hombres) frente al $1 \%$ de la serie general, pero los ingresos por IAVIS han supuesto el $61 \%$ de los ingresos en UVI por IA . No hubo fallecimientos. Un $20 \%$ no pertenecían al área de influencia del hospital.

-IAVE: 49,6\%ooo 319 casos, $70 \%$ no bebedores. Edad y género: Es una IA típicamente masculina (20) (72\%) y significativamente $(\mathrm{p}<0,001)$ diferente del Censo (41). Su número disminuye un $15 \%$ con respecto a nuestra última serie (6) disminución que venía ocurriendo desde 1997(4) con una pérdida acumulada de 103 casos (30\%). La mejor asistencia prehospitalaria, seguramente justifica la reducción de las IAVE hospitalarias ya que según el trabajo de Arnal y cols. (51) sólo trasladan a un centro hospitalario al 42,6\% de los atendidos por SAMUR-PC Este descenso se viene produciendo por reducción de varones, mientras que los casos en mujeres se mantienen, aunque no significativa. Las medias de edad no difieren de la serie general, pero los hombres son ahora algo más jóvenes, con lo que las cifras se sitúan por debajo de 1997 (4) y 2000 (6). Al igual que ocurría con el número, también se mantiene la edad en mujeres, que son tradicionalmente mas jóvenes que los hombres en la IAVE. Desde 1994 (4) el número de IAVE en el grupo de edad más joven disminuye progresivamente en hombres, mientras que aumenta en mujeres. Se han recogido 5 casos de alcohólicos menores de 25 años sin diferencia de género, edad temprana para lo que se considera habitualmente (52), quizás por la influencia del "botellón". En el grupo de alcohólicos encontramos menos casos de depresión, ansiedad, drogadicción e intentos previos de suicidio que entre los no alcohólicos pero la diferencia no es significativa y esto parece habitual $(53,54)$. No hubo fallecimiento ni tampoco ingresos en UVI. La gravedad de los casos lógicamente es mayor con el mejor filtrado prehospitalario (51) pero este tratamiento extrahospitalarios también mejora el resultado de la Urgencia. Rechazaron asistencia prehospitalaria urgente un $21 \%$ (51) y en muestro Centro un $12 \%$ fueron altas voluntarias. Una vez más hay diferencia numérica, no estadística, en la frecuentación según los días y en la comparación de alcohólicos y no alcohólicos

-IAVD: 34\%ooo habitantes. En el año 2004 duplican las del 2000 (6) y superan en cuatro veces las de 1994 (4) y casi alcanzan los datos de 1990 (3) cuando el "boom" de la heroína $(21,33)$ en España. Han pasado de ser el $2 \%$ de las IAV en el 79 (1) o $12 \%$ en el 2000 (6) al $15 \%$ de todas las voluntarias. Es conocido el elevado consumo de drogas en nuestro país, aparentemente el mayor de Europa. Edad y género: La edad de las mujeres es significativamente menor que en las restantes IA. (1-6). Al igual que las IAVE las intoxicaciones agudas 
por drogas son de predominio masculino, crece un $100 \%$ con respecto a los valores de 2000 (6) (167 frente a 83) y parece concluir el mayor crecimiento del género femenino de años anteriores, aunque la evolución en estos 25 años ha sido 2; 42;13;19;33;52 casos. Tóxicos: La heroína ha sido la causa de las $2 / 3$ de los ingresos por drogas en urgencias. En la publicación anterior (6) pronosticábamos el aumento de la MDMA y en efecto, ha pasado de cinco casos en 1997 (5) y catorce en el 2000 (6) a los veintidós actuales. Aparecen nuevas drogas, como el pegamento, sin duda efecto de la inmigración, que también aporta sus tóxicos; la GHB, éxtasis líquido o ketamina ya descrita en Barcelona por Espinosa et al en el 2001 (57) de la que hasta ahora no habíamos recogido ningún caso, por lo que debe haber diferencias locales. Cuatro casos no pudieron ser etiquetados por la dispersión de contenidos de las pastillas ilícitas. La media de tóxicos consumidos en las IAVD fue de 1.8 lo que supone que casi todos los pacientes habían mezclado más de un elemento (40 alcohol, 16 medicamentos y $20 \%$ mezclas de drogas). Algo más de un tercio de los pacientes intoxicados reconocieron ser adictos a las drogas (43\% si sumamos drogas y alcohol) y el resto eran consumidores accidentales o negaban su adicción, esta misma proporción se ha encontrado en las IAVE por lo que aproximadamente dos tercios de los que se intoxican con alcohol o drogas serían consumidores más o menos recientes y por tanto toxicómanos potenciales. La valoración psiquiátrica permitió encontrar nueve casos de depresión y un intento previo de suicidio. El destino de los pacientes intoxicados por drogas fue: alta previa valoración psiquiátrica $15 \%$ y tres ingresaron en UVI, por lo que las drogas causaron el $17 \%$ de los ingresos en UCI. 23 casos alta voluntaria sin concluir estudio, situación bien conocida en las salas de urgencias y en la asistencia prehopitalaria

-IAVSD 14\%ooo: En el año 2004 hay 92 casos, su número crece cada año y así en 1997 (5) fueron 37 y 55 en el 2000 (6) pero este crecimiento es menor que el de la serie general de modo significativo. Es un colectivo en el que predominan las mujeres pero sin diferencia significativa con respecto al grupo Censo $\chi^{2}$ y sí de las IAVIS $\chi^{2}$ de las que se diferencia. Las edades medias son algo superiores a la media general y hay casos desde los 15 a los 97 años. Los tóxicos que las causan son ansiolíticos en las dos terceras partes, analgésicos $11 \%$ y un grupo de varios $16 \%$. Destacar trece pacientes que sobredosificaron antidepresivos y 15 que añadieron alcohol a sus medicamentos. Se solicitó valoración psiquiátrica en un tercio encontrando 28 casos de depresión. En este colectivo había también siete alcohólicos, 7 drogadictos, cuatro demencias y psicopatías y otras tantas enfermedades orgánicas como EPOC, gota o hipertiroidismo. Un paciente precisó ingreso en UVI. Las IAVSD tiene características propias y no se asemejan a las restantes IAV ni a las IAA y es frecuente que haya variaciones poco explicables en las series que componen VEIA Este grupo quizás refleje la menor tolerancia de las personas al "malestar" (el insomnio ha sido el síntoma más habitual a resolver) ;pero también el desconocimiento de los fármacos como en el caso que se sobredosificó heparina o bien los que mezclan medicamentos para potenciar su efecto $(1,4$ de promedio, 1,9 en los IS)

-IAA: representan solo el $17 \%$ del total de la serie con 262 casos $41 \%$ ooo. Un paciente de cada tipo de accidente precisó ingreso en UVI. La mayoría (78\%) son accidentes domésticos. Es habitual el predominio femenino en este tipo de IA pero en esta ocasión la diferencia no es significativa. Y tampoco es significativa la diferencia al comparar el reparto de edades aunque sea apreciablemente mayor a las de las IAV -media $44 \pm 19$ frente a $35 \pm 14$ las voluntarias.

-IAAD: accidentes domésticos 205 de los cuales 136 son mujeres y esa diferencia es significativa con respecto a la serie general. Existe una distribución de los casos con respecto a la edad bimodal como ya hemos descrito con anterioridad (3-5). Gases y cáusticos son la mayoría quedando un $25 \%$ que se reparte entre medicamentos, alcohol y un resto muy variado.

-IAAL: se atendieron 47 hombres y 10 mujeres, la mayoría por gases de combustión y cáusticos. Son más jóvenes que los pacientes por accidentes domésticos.

Queda por ultimo un grupo de 6 pacientes que no hemos podido etiquetar correctamente dentro de los epígrafes anteriores Uno de ellos es un "body packet" al que no podemos identificar como voluntario ni accidental exclusivamente, nosotros no habíamos tenido ningún caso por que estas personas una vez detectadas en la aduana de Madrid son llevadas al HGM que es el hospital de referencia Villalba et al han publicado su dilatada experiencia de 445 casos (58) Este caso podría hacer pensar que existe trafico de este modo también para movimientos dentro del país. Un segundo caso se produjo al ingerir un preso unas pilas para poder salir de la cárcel, a otro en un atraco le obligaron tomar metadona y a otro le dieron una benzodiacepina sin que lo supiera también con fines delictivos. Los dos restantes tomaron por error medicamentos o dosis equivocadas.

\section{Bibliografía}

1. Caballero PJ, Dorado MS, Alonso F. Intoxicación aguda: estudio de 673 casos. Med Clin (Barc) 1981; 77: 139-45.

2. Caballero PJ, Gutiérrez F, Dorado MS. Epidemiología de la intoxicación aguda: Estudio comparativo 1979-1985 en el área sur de la Comunidad Autónoma de Madrid. Rev Clin Esp 1987; 181: 334-9.

3. Dorado MS, Álvarez R, Caballero PJ, Medina J, Casanova C, Granado JA. Epidemiología de la intoxicación aguda: Estudio de 815 casos habidos en 1990 en el área sur de la Comunidad de Madrid. Rev Clin Esp 1992; 191: 131-6.

4. Dorado MS, Martín J, Sabugal G, Caballero PJ. Epidemiología de la intoxicación aguda: estudio de 613 casos habidos en 1994 en el área sur de la Comunidad de Madrid. Rev Clin Esp 1996; 196: 150-6.

5. Caballero PJ, Dorado MS, Brusínt B, Jerez B, Medina M. Vigilancia

epidemiológica de la intoxicación aguda 1997 (estudio de 1.140 casos del área sur de la Comunidad de Madrid). Rev Clin Esp 1999; 199: 42430 .

6. Caballero PJ, Dorado MS, Jerez B, Medina M. Brusínt B. Vigilancia epidemiológica de la intoxicación aguda en el área sur de la comunidad de Madrid: Estudio Veia 2.000 An Med Interna 2004; 21 (2): 62-8.

7. Dorado S. Variaciones epidemiológicas en las intoxicaciones agudas voluntarias atendidas en el hospital Doce de Octubre de Madrid desde 1979-1994. Tesis doctoral. Universidad Complutense de Madrid; 1998.

8. Muñoz J y Guerrero JE. Intoxicaciones: panorama actual: Rev Clin Esp 1996; 196: 141-2.

9. McEvedy CJ. Trends in self-poisoning: Admissions to a central London hospital, 1991-1994. J R Soc Med 1997: 496-8. 
10. Settimi L, Davanzo F, Carbone P, Sesana F, Locatelli C, Farina ML, et al. Surveillance of toxic exposures: the pilot experience of the Poison Control Centers of Milan, Pavia and Bergamo in 2006. Ann Ist Super Sanita 2007; 43: 287-94.

11. Bialas MC, Reid PG, Beck P, et al. Changing patterns of self-poisoning in a UK health district. Q J M 1996; 89: 893-901.

12. Schmidtke A. Bille-Brahe U. De Leo, et al. Attempted suicide in Europe: Rates, trends and sociodemographic characteristic of suicide attempters during the period 1989-1992. Results of the WHO/EURO Multicentre Study on parasuicide. Acta Psychiatr. Scand 1996; 93: 327-38.

13. Kelly CB, Weir J, Rafferty T, Galloway R. Deliberate self-poisoning presenting at a rural hospital in Northern Ireland 1976-1996: relationship to prescribing. Eur Psychiatry 2000; 15: 348-53.

14. Carvajal A, Bachiller A, Rallo R, García L. Intoxicaciones agudas en Valladolid. Estudio epidemiológico de los casos atendidos en el Hospital Clínico desde 1978 a 1984. Med Clin (Barc) 1987; 88: 135-9.

15. Tejedor MC, Díaz AM, Álvarez E, Castillón JJ, Pericay JM. Intento de suicidio: Cambios epidemiológicos entre 1969 y 1996. Estudio retrospectivo de 1.150 casos. Actas Esp Psiquiatr 1999; 27: 292-7.

16. Rygnestad $\mathrm{T}$ y Faberhaug $\mathrm{O}$. Acute deliberate self-poisonings in the area of Trondheim 1978-2002 Tidsskr Nor Laegeforen 2004; 124: 2736-9.

17. Dieserud G, Loeb M, Ekeberg O. Suicidal behaviour in the municipality of Baerum, Norway: A 12-year prospective study of parasuicide and suicide. Suicide Life Threat Behav 2000; 30: 61-73.

18. Rodríguez M A, Barrio G, De la Fuente L, et al. Urgencias relacionadas con el consumo de drogas, alucinógenos y anfetaminas atendidas en quince hospitales españoles durante 1994. Rev Clin Esp 1997; 197: 804-9.

19. Caballero PJ, Dorado MS. Factores epidemiológicos de la intoxicación. Medicine (Madr) 1980; 59: 3691-9.

20. Woolfenden S, Dossetor D, Williams K. Children and adolescents with acute alcohol intoxication/self-poisoning presenting to the emergency department. Arch Pediatr Adolesc Med 2002; 156: 345-8.

21. Crombie IK, McLoone P. Does the availability of prescribed drugs affect rates of self poisoning? Br J Gen Pract 1998; 433: 1505-6.

22. Carlsten A, Allebeck P, Brant L. Are suicide rates in Sweden associated with changes in the prescribing of medicines? Acta Psychiatr Scand 1996; 94: 94-100.

23. Fleischmann A bertolote JM, De Leo D, Phillips M, Sisask M, Vijayakumar L, et al Characteristics of attempted suicides seen in emergencycare settings of general hospitals in eight low- and middle-income countries. Psychol Med 2005; 35: 1467-74.

24. Mairata S, Morey A, Ladaria A, Ballesteros JA, De la Calle F. Intoxicación medicamentosa aguda voluntaria. Estudio epidemiológico en Mallorca. Rev Clin Esp 1981; 163: 307-10.

25. Palop R, Morales FJ, Rodríguez C, Esplugues J. Intoxicaciones agudas y reacciones alérgicas medicamentosas. Estudio de 532 casos. Med Clin (Barc) 1984; 82: 651-55.

26. Bundó M, Fandós JM, Diez J, Ferrán M, Mundet X, Ibáñez L. Intoxicación voluntaria farmacológica en Barcelona: 110 casos. Atención Primaria 1987; 4: 402-6.

27. Fuentes J, García-Galán A, Muñoz JR, et al. Intoxicaciones agudas en la unidad de urgencias de un hospital general. Arch. Fac Med Zaragoza 1990; 1: 27-30.

28. Pascual A, Fuentes F, Castellano M, Ferrer A, López A. Estudio epidemiológico de las intoxicaciones agudas en la población de Zaragoza. An Med Interna (Madrid) 1992; 9: 381-5.

29. Arruga C. Almirall J, Bassa J Balanzó X. Intoxicaciones agudas en un hospital comarcal. Med Clin (Barc) 1992; 98: 117-8.

30. Cabo M, Marti JB, Miralles S, Marti JL. Etiology of intoxication: A study of 557 cases. Eur J Epidemiol 1993; 4: 361-7.

31. Bajo A, Santos ME, Sanz F, et al. Estudio epidemiológico sobre intoxicaciones agudas y dotaciones de botiquines de antídotos. An Med Interna (Madrid) 1999; 16: 285-9.

32. Carpintero JM, Ochoa FJ, Ruiz JI, Bragado L, Palacios G, RamalleGómara E. Prevalencia de las intoxicaciones agudas en Urgencias de La Rioja. Emergencias 2000; 12: 92-7.

33. Palazón C, Segura J, Renedo A, Palazón EL, Pardo JC, Felices F. Intoxicaciones agudas graves en un Servicio de Medicina Intensiva 19861997. Rev Esp Salud Pública 2000; 74 (1).

34. Roca de Togores A, Rubio P, Sánchez P, Rodríguez D, Villar A. Inten- tos de suicidio atendidos en un centro de salud. Atención Primaria 2002; 29: 547-52.

35. Bugarín RR, Galego P, Fernández JL, Mariño AS Consideraciones acerca del tratamientode las intoxicaciones agudas en Atención Primaria. MEDIFAM 2002; 12: 247-59.

36. Marín Lozano, et al. Tentativas de suicidio en la urgencia del hospital general: un estudio epidemiológico y clínico. An Psiquiatría (Madrid) 1995; 1: 259-63.

37. Duce S, López E, Navas V, et al. Intoxicaciones medicamentosas voluntarias atendidas en un Servicio de Urgencias. Emergencias 1998; 10: 225-33.

38. Bugarin R, Galego P, Gude F, García A, Galban C. Estudio de las intoxicaciones etílicas agudas en un servicio de urgencias. An Med Interna (Madrid) 2000; 17: 588-91

39. Carpintero JM, Ochoa FJ, Ruiz JI, Bragado L, Palacios G, RamalleGómara E, et al. Prevalencia de las intoxicaciones agudas en Urgencias de La Rioja. Emergencias 2000; 12: 92-7.

40. Riquelme A, Burillo-Putze G, Jiménez A, Hardisson A. Epidemiología global de la intoxicación aguda en un Área de Salud. Aten Primaria 2001; 28: 506-6.

41. Anónimo: Memoria 2004. Edita Hospital Universitario 12 de Octubre. www.h12o.es

42. Lardeur JY, Compain C, Baudier D, Baudier: Régulation et prise en charge des intoxications volontaires par un service d'aide médicale d'urgence (Pre-hospital emergency management of self-poisoning). Press Med 2001; 30: 626-30.

43. Palazzolo J, Favre P, Julerot JM, Bougerot T. Caractéristiques des patients hospitalisés dans un centre hospitalier spécialisé après une tentative de suicide (About volunteer medicinal intoxication admitted in specialized hospital): Encephale 2002 28: 39-50.

44. Beck P, Lazarus J, Scorer R, Smith P, Routledge P. Increasing rate of deliberate self poisoning. BMJ 1994: 308: 789.

45. Rayón P, Montero D, Santamaría B, Madurga M, De Abajo FJ. Benzodiazepine consumption in Spain. Eur J Clin Pharmacol 1997; 52: 321-3.

46. Mcloone $\mathrm{P}$, Crombie IK. Hospitalisation for deliberate self-poisoning in Scotland from 1981 to 1993 : Trends in rates and types of drug used. $\mathrm{Br}$ J Psychiatr 1996; 169: 81-5.

47. Carlsten A, Waern M, Allebeck P. Suicides by drug poisoning among the elderly in Sweden 1969-1996. Soc Psychiatry Psychiatr Epidemiol 1999: 609-14.

48. Blanc PD, Jones MR, Olson KR. Surveillance of poisoning and drug overdose through hospital discharge coding, poison control center reporting, and the Drug Abuse Warning Network. Am J Emerg Med 1993; 11: 14-9.

49. Hidalgo Rodrigo MI, Santiago Guervos M, García Serna B, González Manjavacas C. Las conductas autoagresivas en el servicio de urgencias. Aten Primaria 1995; 15: 412-3.

50. Verstraete AG y Buylaert WA. Survey of patients whith acute poisoning seen in the Emergency Department of the University Hospital of Gent between 1983 and 1990 Eur. J Emerg Med 1995; 4: 217-23.

51. Arnal D, Bernardino M, León JA, Velayos C, Gómez JC, Gilarranz JL. Atención por intoxicación etílica en un Servicio de Emergencia Médica prehospitalario en Madrid. Emergencias 1998; 10: 381-7.

52. Del Río Mc. Consumo de alcohol y problemas relacionados con el alcohol en España. Jano 1998; 54: 197-203

53. Sher L. Risk and protective factors for suicide in patients with alcoholism. ScientificWorld J 2006; 6: 1405-11.

54. Soukas J, Lönnqvist J. Suicide attempts in which alcohol is involved: A special group in general hospital emergency rooms. Acta Psychiatr. Scand 1995: 91: 36-40.

55. Carter GL, Whyte IM, Ball K, et al. Repetition of deliberate self-poisoning in an Australian hospital-treated population. Med J Aust 1999; 170: 307-11.

56. Kapur N, House A, Creed F, Feldman E, Friedman T, Guthrie E. General hospital services for deliberate self-poisoning: An expensive road to nowhere? Postgrad Med J 1999; 888: 599-602.

57. Espinosa G, Miró O. Intoxicación por éxtasis líquido: estudio de 22 casos. Med Clin (Barc) 2001; 117: 56-8.

58. Gómez Antúnez, Cuenca C, Farfán A, Villalba MV, Del Toro J, García J. Complicaciones en transportadores intestinales de paquetes con cocaína. Estudio de 215 casos. Med Clin (Barc) 1998; 111: 336-7. 